\title{
The use of the external layer of the calvaria's frontal bone to repair craniofacial skeleton injuries in Macaca mulatta (Rhesus) ${ }^{1}$
}

\author{
José Mário Camelo-Nunes² \\ Ubimara Pereira Rodrigues $^{3}$ \\ Saul Goldenberg ${ }^{4}$
}

\begin{abstract}
Camelo-Nunes JM, Rodrigues UP, Goldenberg S. The use of the external layer of the calvaria's frontal bone to repair craniofacial skeleton injuries in Macaca mulatta (Rhesus). Acta Cir Bras [serial online] 2003 Mar-Apr;18(2). Available from URL: http://www.scielo.br/acb.
\end{abstract}

\begin{abstract}
Purpose: To study the integration of the external layer of the calvaria's frontal bone to repair craniofacial skeleton injuries in primates. Methods: Ten Rhesus monkeys underwent surgery in two stages. In the first stage, four bone fragments were harvested from the external layer of the calvaria's frontal bone and were transplanted two on the calvaria's frontal bone and the other two onto the maxillary bone, by onlay and inlay. Seven weeks thereafter, four fragments were extracted from the transplantated area. The animals were not sacrificed. Results: Macroscopic examination reveals bone regeneration, the areas onto which the bone fragments were transplantated having consequently increased in volume. The results of optical and electron microscopy is being carried out.
\end{abstract}

KEY WORDS - Skull. Bone transplantation. Macaca mulatta.

\section{Introduction}

The use of the calvaria's frontal bone as a source of bone donating tissue for the treatment of craniofacial skeleton deformities (congenital and acquired) was popularized in 1982, by Tessier ${ }^{(1)}$.

In humans, these transplants are generally harvested from the parietal bone, specifically from its external layer, which allows the cranial cavity to remain intact ${ }^{(2-6)}$.

In our review of the pertinent literature found no research on the use of the external layer of the calvaria's frontal bone to repair craniofacial skeleton injuries of non-human primates.

\section{Methods}

\section{Sample}

Consisted of ten mammals of the Primate order, Catarrhini infraorder (Old World monkeys), Cercophitecos family, Macaca genus, and Mulatta species (Rhesus).

In January 1999 the birth of ten animals was programmed. These were specifically used for this study.

The sample consisted on seven male monkeys and three female. The animals weight were between $1992,0 \mathrm{~g}$ to $3128,0 \mathrm{~g}$ and 19 to 23 months old.

1 Work performed in the Department of Plastic Surgery of the Medical School Fundação ABC.

2 Professor of Plastic Surgery of the Medical School of the Fundação ABC - São Paulo, Brazil.

3 Technical Director of the Central Biotic Resources Division of the Instituto Butantan - São Paulo, Brazil.

4 Full Professor Department of Surgery Federal University of São Paulo. Chief Editor of Acta Cirúrgica Brasileira. 
The experimental protocol was approved by the Animal Research Ethics Comittee of the Medical School of the Fundação ABC, São Paulo - Brazil.

The monkeys were severely controlled with regard to both ecto- and endo-parasites, tuberculinization, dwelling, physical and chemical restraint, feeding, water supply, fluid extraction, mating, and control of growth and development (7).

\section{Procedures}

The animals were submitted to pre-surgical tests (hemogram, coagulation profile and glycemia), were considered healthy and made available for the surgical procedures. All underwent a two-hour pre-surgical fast.

Simple $x$-rays of the head, fontal and profile positions, and of the closed maxilla and mandible were taken and moulds of the dental arcade were cast in order to produce gypsum models. All stages of research (including pre- and post-surgery) were photographed, both conventionally and digitally, and filmed.

The animals were given $0.3 \mathrm{mg} / \mathrm{Kg}$ of a cetamine hydrochloride solution and $7.5 \mathrm{mg} / \mathrm{Kg}$ of 2-(2.6 xilidine)-5,6-dihydro-4H-1,3-tiazine intramuscularly as pre-anaesthesic medication.

They were considered to be pre-anaesthetized on average after four minutes, by which time they had lost their aggressiveness, were in a lateral decubitus position, open-eyed and with miotic pupils.

Anaesthesia was controlled by means of pulmonary and cardiac auscultation, peripheral blood oxygen saturation, peripheral pulse count and observation of pupil reflexes and eyelid movement.
Anaesthesia was maintained with cetamine hydrochloride, an average of 2.25 doses of $16.75 \mathrm{mg} / \mathrm{Kg}$ each having been used at average intervals of 58.25 minutes.

Local anaesthesia of the scalp and of the oral cavity was applied through the infiltration of a solution with bupivacaine hydrochloride $(5 \mathrm{mg} / \mathrm{ml})$, lidocain hydrochloride (20 mg/ml) and epinephrine with a final concentration of 1:400,000.

The monkeys were placed on the surgical table in a horizontal dorsal decubitus position, the head partially flexed with the help of a pad. The upper limbs were maintained in an abducted position, by bandaging and the lower limbs were extended and immobilized in the same way; the sensor for peripheral monitoring was attached to the left foot.

The surgical team consisted of one surgeon, one anaesthetist, two assistants and one surgical technologist handling the instrumentation.

The study was divided into two surgical stages. In the first intervention four calvaria's bone fragments were harvested from the external layer of the calvaria's frontal bone. Two of them were transplantated onto the calvaria's frontal bone (one on the same donating place - onlay and the other on the calvaria's frontal bone - inlay) and the other two onto the maxilla (one defected by means of bone wear away - onlay and the other on the maxilla - inlay) and fixed with titanium microscrews (Osteomed ${ }^{\mathrm{TM}}$ ).

Seven weeks later, four fragments were removed from the aforementioned transplants. All the fragments were measured with digital calipers accurate to the thousandth of a millimeter as regards their vertical and horizontal dimensions, as well as their thickness. 


\section{Preliminary Results}

Preliminary macroscopic results show bone regeneration with the volume of the facial areas

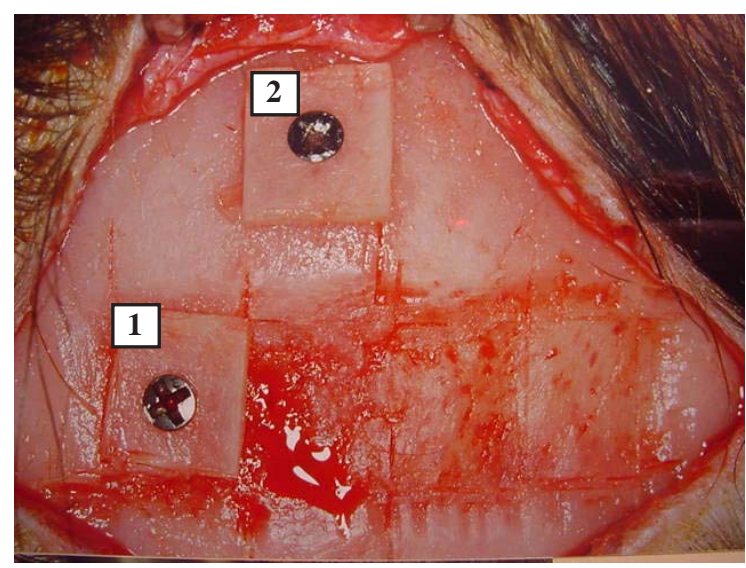

FIGURE 1 - Calvaria ( $1^{\text {st }}$ intervention); donor areas and positioning of the transplants 1 and 2, with rigid fixation.

FIGURA 1 - Calvária ( $1^{2}$ intervenção), áreas doadoras e posicionamento dos transplantes 1 e 2, com fixação rígida.

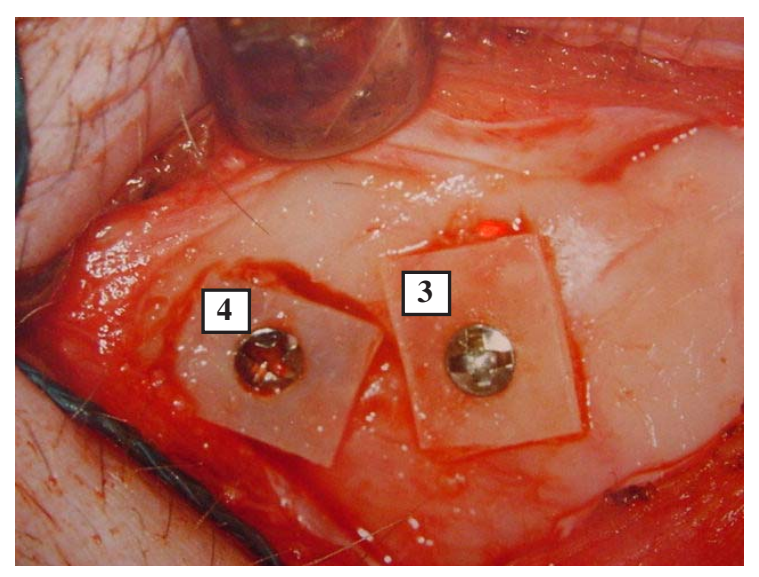

FIGURE 3 - Maxillary bone ( $1^{\text {st }}$ intervention), positioning of the transplants 3 and 4.

FIGURA 3 - Osso maxilar ( $1^{\mathrm{a}}$ intervenção), posicionamento dos transplantes 3 e 4, com fixação rígida.

Microscopic study involving optical microscopy and electron micrography is currently being carried out.

No complications arose during the postsurgical period and all the animals were monitored on a daily basis and analyzed, under anaesthesia, 21 days after each of the two interventions. The animals are currently back in their natural habitat.

The data and illustrations are in the process of being recorded in the respective protocols for subsequent publishing. onto which the bone fragments were grafted having consequently increased (Figures 1, 2, 3 and 4).

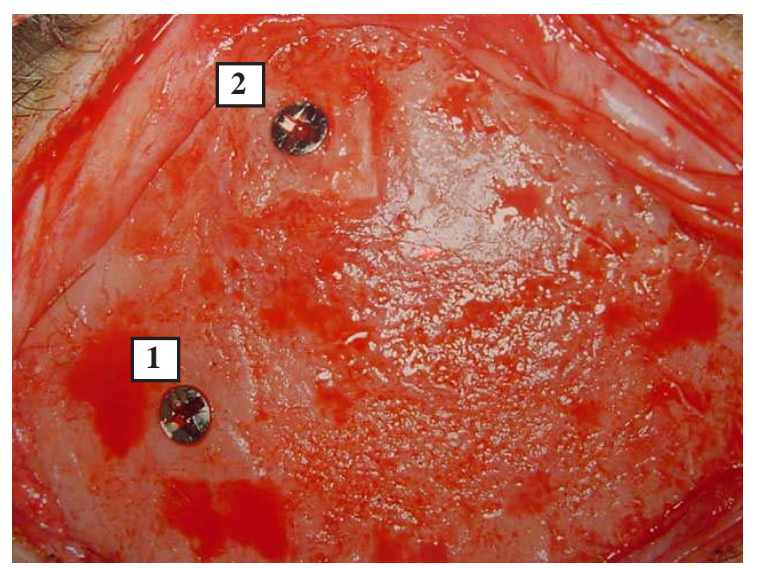

FIGURE 2 - Calvaria (2 ${ }^{\text {nd }}$ intervention), seven weeks later, transplants positions 1 and 2 .

FIGURA 2 - Calvária ( $2^{a}$ intervenção), após 7 semanas, local dos transplantes 1 e 2 .

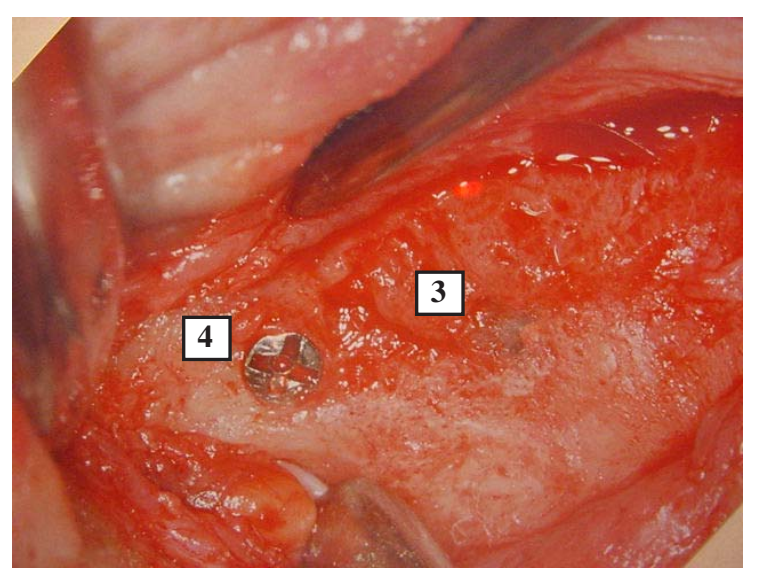

FIGURE 4 - Maxillary bone (2nd intervention ), seven weeks later, transplants positions 3 and 4.

FIGURA 4 - Osso maxilar ( $2^{\text {a }}$ intervenção), após 7 semanas, local dos transplantes 3 e 4 .

\section{References}

1. Tessier P. Autogenous bone grafts taken from the calvarium for facial and cranial applications. Clin Plast Surg 1982; 9: 531-8.

2. Kline RM, Wolfe SA. Complications associated with the harvesting of cranial bone grafts. Plast Reconstr Surg 1995; 95: 5-20.

3. Tulasne JF, Camelo-Nunez JM. Greffe osseuse crânienne intra-sinusienne. J de parondontologie \& d'implantologie orale 1999; 18: 183-97.

4. Camelo-Nunes JM, Pereira Filho GV, Spada Neto E, Dall’Olio G. Enxerto ósseo craniano intra-sinusiano. 
Arq Med ABC 2001; 24: 60-9.

5. Ishizuka MMA. Contribuição ao estudo da espessura da calota craniana [thesis]. São Paulo: Universidade Federal de São Paulo; 1987.

6. Gomes Filho, WR. Espessura do enxerto de calota craniana e crista ilíaca alveolar da maxila como base nos implantes em titânio [thesis]. São Paulo: Universidade Federal de São Paulo; 2001.

7. Sanitary control program of the primate (rhesus) colony of the Instituto Butantan, São Paulo, 2002.

\section{Acknowledgements}

Andressa M. C. Inai, Carlos A. Andreucci, Cassio M. R. Amaral, Dirceu Solé, Eduardo F. Simões, Elie Fiss, Elizabeth J. G. Valentini, Ercole S. Neto, Eric Solyom, Fernão S. Oliveira, Gerson V. P. Filho, Giancarlo Dall’Olio, Hisakazu Hayashi, Jean-François Tulasne, Juliana B. Garcia, Ligia M. C. Nunes, Neil F. Novo, Osvaldo Mora, Paul Tessier, Paulo O. Gomes, Renato Albieri, Saul Goldenberg, Teresa R. B. Adão, Vânia G. M. Mattaraia.

\title{
SAME ARTICLE IN PORTUGUESE LANGUAGE MESMO ARTIGO NO IDIOMA PORTUGUÊS
}

2 - NOTA PRÉVIA

\section{Emprego da lâmina externa da região frontal da calvária, na reparação de lesões do esqueleto craniofacial em Macaaca mulatta (Rhesus) ${ }^{1}$}

\section{José Mário Camelo-Nunes ${ }^{2}$ \\ Ubimara Pereira Rodrigues $^{3}$ Saul Goldenberg ${ }^{4}$}

\begin{abstract}
Camelo-Nunes JM, Rodrigues UP, Goldenberg S. Emprego da lâmina externa da região frontal da calvária na reparação de lesões do esqueleto craniofacial em Macaca mulatta (Rhesus). Acta Cir Bras [serial online] 2003 Mar-Abr;18(2). Disponível em URL: http://www.scielo.br/acb.

RESUMO - Objetivo: Estudar a integração da lâmina externa da calvária, na reparação de lesões do esqueleto craniofacial em primatas. Métodos: Foram operados dez macacos Rhesus em duas etapas. Na primeira foram retirados quatro fragmentos ósseos da lâmina externa da região frontal da calvária e transplantados, dois na calvária e dois no osso maxilar por aposição e reposição. Sete semanas após, foram retirados quatro fragmentos dos locais transplantados. Os animais não foram sacrificados. Resultados: Macroscopicamente evidenciou-se regeneração óssea, com conseqüente aumento de volume dos leitos receptores na face. Aguarda-se análise da microscopia óptica e eletrônica.
\end{abstract}

DESCRITORES - Crânio. Transplante ósseo. Macaca mulatta.

\section{Introdução}

A utilização da calvária como tecido ósseo doador para o tratamento das deformidades (congênitas e adquiridas) do esqueleto craniofacial foi popularizada em 1982, por Tessier ${ }^{(1)}$.

Em geral, no ser humano, esses transplantes são obtidos a partir do osso parietal, mais especificamente de sua lâmina externa o que permite a manutenção da integridade da cavidade craniana $^{(2-6)}$

Na busca da literatura não foram encontrados trabalhos de pesquisa referentes ao emprego da lâmina externa da região frontal da calvária, na reparação de lesões do esqueleto craniofacial em primatas não humanos.

\section{Métodos}

\section{Amostra}

Foram utilizados dez mamíferos da ordem Primata, infra-ordem Catarrhini (macacos do velho mundo), família Cercophitecos, gênero Macaca e espécie Mulatta (Rhesus).

Em janeiro de 1999 foi programado o nascimento de dez animais, os quais foram 
utilizados, especificamente, para este estudo.

A população foi constituída de sete macacos machos e três fêmeas. $\mathrm{O}$ peso dos animais variou de $1992,0 \mathrm{~g}$ a 3128,0g e a idade de 19 a 23 meses.

O protocolo experimental foi aprovado pelo Comitê de Ética em Experimentação Animal da Faculdade de Medicina da Fundação ABC.

Os animais foram submetidos a rigoroso controle de ecto e endoparasitas, tuberculinização, alojamento, contenção física e química, alimentação, fornecimento de água, extração de fluídos, acasalamento e controle de crescimento e desenvolvimento ${ }^{(7)}$.

\section{Procedimentos}

Os macacos foram submetidos a exames préoperatórios (hemograma, coagulograma e glicemia), considerados hígidos e liberados para o procedimento cirúrgico. Todos foram mantidos em jejum de duas horas.

Foram realizados radiografias simples da cabeça nas posições póstero-anterior e perfil, da maxila e mandíbula em oclusão, moldes das arcadas dentárias para confecção de modelos em gesso e fotografias (papel e digital) além de filmagem, no pré e pósoperatório de todas as etapas da pesquisa.

Os animais receberam solução de cloridrato de cetamina e 2-(2,6-xilidino)-5,6-dihidro-4H-1,3-tiazina como medicação pré-anestésica via intramuscular nas doses de 0,3 mg/Kg e 7,5 mg/Kg, respectivamente.

Em média, após quatro minutos, foram considerados anestesiados, quando perderam a agressividade, permaneceram em decúbito lateral, mantiveram as pálpebras abertas e as pupilas em miose.

A anestesia foi controlada mediante ausculta pulmonar e cardíaca, contagem do pulso periférico, observação de reflexos pupilares, movimentos palpebrais e controle da saturação periférica de oxigênio.

A manutenção da anestesia foi realizada com cloridrato de cetamina, com média de 2,25 doses de $16,7 \mathrm{mg} / \mathrm{Kg}$ cada, em intervalos em média de 58,25 minutos.

A anestesia local do couro cabeludo e da cavidade oral foram realizadas por meio de infiltração de solução contendo cloridrato de bupivacaína $5 \mathrm{mg} / \mathrm{ml}$, cloridrato de lidocaína $20 \mathrm{mg} / \mathrm{ml}$ e epinefrina com uma concentração final de 1:400.000.

Os macacos foram posicionados na mesa operatória em decúbito dorsal horizontal, com a cabeça sobre um coxim. Os membros superiores foram mantidos em abdução por enfaixamento e os membros inferiores foram mantidos em extensão, sendo que foi fixado no pé esquerdo o sensor para monitorização periférica.

A equipe de cirurgia foi composta por: um cirurgião, anestesiologista, dois auxiliares e instrumentador.

O estudo foi realizado em duas etapas operatórias. Na primeira intervenção foram retirados quatro fragmentos ósseos da lâmina externa do osso frontal da calvária sendo transplantados dois na calvária (um no mesmo local doador - reposição e outro sobre a calvária - aposição) dois no osso maxilar (um em defeito criado por desgaste ósseo - reposição e outro sobre a maxila - aposição), por meio de fixação rígida com microparafusos de titânio (osteomed $®$ ).

Sete semanas após, foram retirados quatro fragmentos dos locais de transplante anteriormente citados. Todos os fragmentos foram aferidos com paquímetro digital, com precisão milesimal nas dimensões vertical, horizontal e espessura.

\section{Resultados Preliminares}

Os resultados macroscópicos preliminares mostram regeneração óssea com conseqüente aumento de volume dos leitos receptores na face (Figuras 1, 2, 3 e 4 ).

O estudo microscópico está sendo realizado mediante microscopia óptica e eletrônica.

O pós-operatório transcorreu sem complicações. Todos os animais foram acompanhados cotidianamente e analisados sob sedação após 21 dias, tanto da primeira como da segunda intervenção e atualmente encontramse e em seu habitat natural.

Os dados e ilustrações estão sendo registrados nos respectivos protocolos, para posterior publicação.

\section{ATENÇÃO: As figuras e as referências estão no texto em inglês.}

Conflito de interesse: nenhum Fonte de financiamento: nenhuma

Correspondência:

José Mário Camelo-Nunes

R. Borges Lagoa, 783/12

04038-031 São Paulo - SP

Tel.: (11)5579-8938

camelonunes@aol.com

Data do recebimento: 10/12/2002

Data da revisão: 14/01/2003

Data da aprovação: 04/02/2003 